\title{
Broadband model-based optoacoustic microscopy enables deep-tissue imaging beyond the acoustic diffraction limit
}

\section{Weiye Li}

University and ETH Zurich

Urs Alexander Tassilo Hofmann

ETH Zurich

Johannes Rebling

https://orcid.org/0000-0003-1336-4359

\section{Quanyu Zhou}

University of Zurich

\section{Zhenyue Chen}

University of Zurich

Ali Özbek

ETH Zurich

\section{Yuxiang Gong}

University and Zurich and ETH Zurich

\section{Daniel Razanksy}

University of Zurich

Xose Luis Dean-Ben ( $\boldsymbol{\nabla}$ xl.deanben@pharma.uzh.ch )

University and ETH Zurich https://orcid.org/0000-0002-8557-7778

\section{Article}

Keywords: Super-resolution, model-based image reconstruction, optoacoustic image formation, intravital microscopy, skin imaging

Posted Date: May 27th, 2021

DOl: https://doi.org/10.21203/rs.3.rs-496385/v1

License: (c) (i) This work is licensed under a Creative Commons Attribution 4.0 International License.

Read Full License 


\title{
Broadband model-based optoacoustic microscopy enables deep-tissue imaging beyond the acoustic diffraction limit
}

\author{
Weiye $\mathrm{Li}^{\dagger}$, Urs A. T. Hofmann ${ }^{\dagger}$, Johannes Rebling, Quanyu Zhou, Zhenyue Chen, Ali Ozbek, Yuxiang Gong, \\ Daniel Razansky, and Xosé Luís Deán-Ben* \\ Institute for Biomedical Engineering and Institute of Pharmacology and Toxicology, University of Zurich \\ and ETH Zurich, Switzerland \\ *Corresponding author: xl.deanben@pharma.uzh.ch \\ ${ }^{\dagger}$ These authors contributed equally to this work
}

\begin{abstract}
Optoacoustic microscopy (OAM) retrieves anatomical and functional contrast in vivo at depths not resolvable with optical microscopy. Recent progress on reconstruction algorithms have further advanced its imaging performance to provide high lateral resolution ultimately limited by acoustic diffraction. In this work, we suggest a new broadband model-based OAM (MB-OAM) framework efficiently exploiting scanning symmetries for an enhanced performance. By capitalizing on the large detection bandwidth of a spherical polyvinylidene difluoride (PVDF) film while accurately accounting for its spatial impulse response, the new approach significantly outperforms standard OAM implementations in terms of contrast and resolution, as validated by functional in vivo experiments in mice and human volunteers. Furthermore, L1-norm regularization enabled resolving structures separated by less than the theoretical diffraction-limited resolution. This unique label-free angiographic performance demonstrates the general applicability of MB-OAM as a super-resolution deep-tissue imaging method capable of breaking through the limits imposed by acoustic diffraction.
\end{abstract}

\section{Keywords}

Super-resolution, model-based image reconstruction, optoacoustic image formation, intravital microscopy, skin imaging

\section{Introduction}

Advanced acoustic inversion methods are essential to optimize the achievable resolution, contrast, and overall performance of optoacoustic (OA, photoacoustic) imaging systems operating at depths not resolvable with optical microscopy ( $>1 \mathrm{~mm}$ in biological tissues). The type and location of ultrasound (US) sensor(s) mainly determine the imaging performance in this depth range, thus are generally tailored for the biomedical application of interest [1, 2]. For example, OA tomography based on concave piezoelectric arrays has been shown to capitalize on a large angular coverage to render accurate images of arbitrarily-oriented vascular networks [3-5]. However, although tomographic microscopic imaging has 
been enabled with high-resolution arrays [6], deep-tissue OA microscopy (OAM) is typically performed by raster-scanning a single-element transducer [7-12]. This approach represents one of the most common OA embodiments and is increasingly being used in preclinical and clinical studies [13, 14]. An important advantage of OAM over tomographic OA methods is a significantly simpler and less expensive instrumentation. More importantly, OAM is particularly suitable for exploiting the multiscale OA imaging capabilities by covering an otherwise unachievable signal bandwidth. For example, centimeter-scale depths have been achieved with transducers featuring detection bandwidths of a few $\mathrm{MHz}[7,15]$, while ultra-wideband detectors have been used e.g. in raster scan OA mesoscopy (RSOM) for high-resolution imaging at depths around $2 \mathrm{~mm}-4 \mathrm{~mm}[13,16]$. Multiple scales can additionally be covered simultaneously e.g. via coaxial light focusing in hybrid-focus OAM $[17,18]$ or with a dual-element transducer in quad-mode OAM [19].

The importance of OAM fostered ongoing efforts on the optimization of image formation methods. The synthetic aperture focusing technique (SAFT), also used in radar, sonar, and biomedical US, has become the standard image formation method in OAM [10]. SAFT was introduced in OAM as the virtual detector concept [20], and is generally implemented using the delay-and-sum (DAS) technique in the time domain [21]. More advanced approaches based on weighting factors in the time or frequency domains $[22,23]$ or on delay-multiply-and-sum (DMAS) methods $[24,25]$ have further been shown to provide an enhanced performance. DAS can be regarded as a discrete implementation of the filtered backprojection algorithm, derived from the time-domain OA forward model for short-pulsed excitation [26]. Such a model represents a solid mathematical foundation enabling the development of more accurate acoustic inversion methods. Thus, algorithms based on time reversal techniques $[27,28]$, model-based (iterative) inversion algorithms [29-31] or hybrid-domain modeling of a focused transducer [28] have been suggested for OAM. Model-based inversion in the time domain is arguably the most accurate approach to account for the finite size and shape of US sensors [32-34]. Additionally, the performance of iterative inversion can be enhanced by properly choosing constraints or regularization terms [35-40]. However, implementation of this method for the dense grid of voxels required for high-resolution volumetric imaging has remained challenging due to extremely high computational costs, even when considering efficient parallel implementations in graphics processing units (GPUs) [41, 42].

Herein, we introduce a new broadband model-based OAM (MB-OAM) image formation framework that efficiently exploits scanning symmetries to enable GPU-based, high-resolution, volumetric imaging. The underlying time-domain OA forward model accurately accounts for the large bandwidth of the OA signals collected with a custom-made spherical polyvinylidene difluoride (PVDF) transducer, while discretization of the transducer surface additionally enables modeling its spatial impulse response. Model-based inversion is shown to significantly enhance the achievable resolution even beyond the acoustic diffraction limit, thus outperforming standard OAM implementations as well as other optical modalities based on extrinsically-administered contrast agents. The label-free nature of OA ensures that the suggested approach is generally applicable in any preclinical or clinical application requiring visualization of tissues at depths not reachable with optical microscopy.

\section{Results}

Accurate modeling of OA signals 
OAM acquires volumetric images in a raster-scan acquisition protocol (Fig. 1a and Suppl. Fig. 1) with spatial sampling (scanning step) fulfilling the Nyquist criterion. The lateral resolution in OAM is considered to be limited by acoustic diffraction to $0.71 \lambda / N A$ [43], where $\lambda$ and NA are the central wavelength and numerical aperture of the US transducer. Typical lateral resolutions are in the range of $40 \mu \mathrm{m}$, for which scanning steps around a few tens of micrometers are needed. The suggested MB-OAM defines time-resolved OA signals corresponding to each pair of voxel and scanning position, where the lateral positions of the voxels match the $x$ - $y$-scanning positions (see methods for a detailed description). For a raster-scan configuration, the OA model is translational-symmetric - signals are identical if the relative position between the voxel and scanning point is maintained (Fig. 1b). A spherical US transducer further defines axial symmetries with respect to the acoustic axis (Fig. 1b). Accurate modeling of the impulse response (IR) of the transducer is achieved by splitting it into two terms, namely the electrical impulse response (EIR) defining the effective detection bandwidth, and the spatial impulse response (SIR) accounting for acoustic diffraction effects associated to the finite size of the sensing area [44]. A custom-made transducer consisting of a PVDF film featuring broad detection bandwidth ( $1 \mathrm{MHz}$ to $100 \mathrm{MHz}$ ) was used herein [11] (Fig. 1c, see methods for a detailed description). However, the spatiallydependent SIR of the spherically-focused, axially-symmetric transducer strongly limits the lateral resolution in out-of-focus regions and standard image formation methods (SAFT and its variants) cannot fully compensate for this effect. In the suggested model-based framework, the SIR for each voxel was estimated by superimposing the corresponding time-resolved OA signals for a set of surface elements ( 1000) covering the active sensing area (Fig. 1c). This is equivalent to adding up the OA forward model corresponding to each surface element (see methods for a detailed description). Considering the scanning symmetries, the information in the model-matrix is fully contained in the sub-matrix corresponding to an individual scanning point, thus could be stored in the memory of state-of-the-art GPUs (1.3 GB for half a million voxels and 837 time points, see methods for details). The memory required to store the $\mathrm{OA}$ forward model is invariant to the arbitrarily-large lateral dimensions of the scan area, which allows storing the OA signals, the model, and the reconstructed absorber distribution simultaneously on a GPU. Thereby, large volumetric images can be reconstructed at high-resolution in a reasonable time ( $\sim 12$ minutes). The amplitude spectral density of the time-domain OA forward model (columns of the model matrix) reveals the expected diffraction patterns for different frequencies, while their amplitude in the time domain defines a transducer sensitivity field matching the expected diffraction-limited resolution for broadband OA signals (Fig. 1d). The capability to encode frequencydependent information empowers the suggested model-based framework with unique capabilities to reconstruct absorbers with different sizes emitting OA waves in different frequency bands, clearly outperforming alternative approaches e.g. based on superimposing signals or SAFT (Fig. 1e).

Imaging beyond the acoustic diffraction barrier

Mathematically, model-based inversion minimizes an energy functional consisting of a data fidelity term driving the solution towards the observed data and a regularization term that stabilizes the inversion and includes prior information [45]. OAM image formation is challenged by the limited-view scanning geometry and the associated ill-posed nature of the inverse problem [46]. Regularization hence turns essential for computing a credible approximation of the light absorption distribution. Tikhonov regularization, based on the $\mathrm{L} 2$ norm, is the classical method for noise-robust inversion and represents the most probable solution given the raw signals and a prior Gaussian distribution of measurement 
noise. The model-based algorithm based on an $\mathrm{L} 2$ regularization term (MB-L2, see methods for details) enabled enhancing the lateral resolution and contrast of OAM images at out-of-focus locations beyond SAFT (Fig. 1e). However, reconstruction of closely separated $(\sim 40 \mu \mathrm{m})$, simulated absorbers at the focal plane indicate that the lateral resolution is still limited by acoustic diffraction (Figs. 2a and 2b). The OA signals and forward model were bandpass filtered between 0.5 and $80 \mathrm{MHz}$, yielding an effective diffraction limit of $61 \mu \mathrm{m}$ (see methods for details). This is expected considering that MB-L2 is a linear inversion procedure equivalent to time-reversed propagation of US waves [47]. Alternatively, regularization terms based on the L1 norm can be used to promote sparse solutions in a given domain. Particularly, a L1-based regularization term in the image domain translates to most voxels having zero (or close to zero) value. Simulation results with relatively separated absorbers indicated that the full width at half maximum (FWHM) of the absorbers reconstructed with the model-based algorithm with L1 regularization (MB-L1, see methods for details) is considerably smaller $(13 \mu \mathrm{m})$ than the FWHM achieved with SAFT and MB-L2 (41 $\mu \mathrm{m}$, Figs. $2 \mathrm{a}$ and $2 \mathrm{~b})$. This could be erroneously interpreted as a narrower point spread function (PSF) of the imaging system enabling higher resolution. However, MB-L1 is a non-linear inversion procedure and hence a PSF cannot be defined. Instead, the achievable resolution must be estimated from the reconstructed image of closely separated sources. In this regard, undistinguishable absorbers in the images obtained with SAFT and with MB-L2 could be separated with MB-L1, thus effectively breaking through the acoustic diffraction barrier (Figs. $2 a$ and $2 b$ and Suppl. Fig. 2). Experimental results with an agar phantom embedding a $\sim 20 \mu \mathrm{m}$ microsphere slightly out-offocus corroborated the simulation results (Figs. $2 \mathrm{c}$ and $2 \mathrm{~d}$, see methods for a detailed description). The FWHM of the reconstructed sphere was significantly reduced with MB-L1 compared to SAFT and MB-L2, while it was also possible to distinguish two closely separated spheres simulated by shifting the scanning position and adding up the corresponding OA signals (Figs. $2 \mathrm{c}$ and $2 \mathrm{~d}$, see methods for a detailed description). Additional experiments with an agar phantom embedding carbon fibers with $7 \mu \mathrm{m}$ diameter further validated the enhanced resolution achieved with MB-L1 (Figs. 2e and 2f). The width of the reconstructed fibers was significantly reduced with MB-L1. More importantly, MB-L1 enabled resolving crossing fibers at points where SAFT and MB-L2 failed (Fig. 2f).

\section{Enhanced angiographic imaging in vivo}

The accuracy of the OA forward model turns essential for precise reconstruction of more complex structures in living organisms. Proper selection of the regularization term is also highly important. Most commonly, OA visualizes multi-scale vascular networks in mammalian tissues with no dominant components in space or frequency domains, which challenges defining information a priori on the images. A raster-scan with $25 \mu \mathrm{m}$ step across the back of a CD-1 mouse (Fig. 3a, see methods for a detailed description) enabled comparing the in vivo imaging performance of different image formation approaches. SAFT, MB-L2, and MB-L1 successfully visualized microvascular structures in this region, although clear differences were observed in image quality and content (Fig. 3b). A smoothing effect was observed in the blood vessels reconstructed with SAFT degrading the achievable resolution. The vascular network at different depths is clearly better resolved in the MB-L2 image, including branches not visible with SAFT. The sharpening effect of MB-L1 compared to MB-L2 was similar to the observations in simulations and phantom experiments. The width of the reconstructed vessels is clearly narrowed compared to the other two approaches. However, MB-L1 appears to reduce the available information in 
the image. Many of the branches visible in the MB-L2 image are not seen with MB-L1, arguably due to the sparsity constraint. The achievable resolution with different approaches can be more clearly compared in a three-dimensional view of the central part of the scan (Fig. 3c). Individual vessels and branches in MB-L1 and MB-L2 images are shown to be more clearly defined than in superimposed MBL2 and SAFT images, respectively. A comparison of the image profiles for a region close to a branching point corroborates that MB-L1 can break through the resolution limit of the other two approaches (Fig. 3d). The respective image content was better assessed by quantifying the amount of vessels in the binarized images with an automatic vessel segmentation and analysis (AVSA) algorithm [48] (Fig. 3e, see methods for a detailed description). Statistical analysis of the number of detected vessels from four different mouse back datasets showed that, on average, the number of vessels in the MB-L2 image is higher by almost two-fold with respect to SAFT and MB-L1 images (Fig. 3f). Overall, the suggested model-based image formation framework significantly enhanced the performance of SAFT in vivo, while the regularization choice establishes a trade-off between achievable resolution and visibility of vascular networks.

\section{Towards quantitative clinical imaging}

Microvascular alterations are involved in many physiological and pathophysiological processes and serve as indicators of diabetes, cancer, neurological disorders, and other human diseases. The powerful microvascular imaging capabilities of the suggested MB-OAM are best appreciated when validating the images obtained against those rendered with established angiographic methods (Fig. 4a). A bright field image of a region in the back of a nude-Fox1nu mouse enables identifying superficial blood vessels within the skin. Vascular contrast and imaging depth can be enhanced e.g. via injection of indocyanine green (ICG), a water-soluble dye routinely used in the clinics that fluoresces in the near infrared (NIR-I, $\sim 650-1000 \mathrm{~nm}$ ) window of light. Recently, the availability of cameras sensitive to photons in a second near-infrared (NIR-II, $\sim 1000-1700 \mathrm{~nm}$ ) window featuring suppressed light scattering and autofluorescence has enabled reaching depths previously unattainable with optical methods. For this, water-soluble quantum dots emitting light in this wavelength range can be used, which are however not approved for clinical use. The vascular structures observed in the fluorescence images are also visible in the image obtained with MB-L2, which additionally enables visualizing smaller and deeper vessels with endogenous contrast (Fig. 4a). The capability of the suggested approach to quantify microvascular changes was further tested by assessing responses to thermal stimuli in images of the cuticle microvasculature of healthy volunteers (see methods for a detailed description). MB-L2 images acquired after exposure to cold and warm water revealed a higher vascular density in the second case (Fig. 4b). Qualitative image quality may further be enhanced via post-processing e.g. with a Frangi filter, which may however lead to the appearance of inexistent vessels in the images (Suppl. Fig. 3). Considering that the tissue temperature is expected to be approximately the same at the measuring time points, these differences are ascribed to thermally-induced microvascular alterations. Quantification of vasodilation was better achieved in MB-L1 images of the nailfold region (Fig. 4c). Numerical simulations considering spheres with different sizes (see methods for details) indicate that MB-L1 provides more accurate dimensional readings than MB-L2 (Fig. 4d, Suppl. Fig. 4). MB-L1 further capitalizes on the relative sparsity of the images of this area to better resolve arterioles and venules. Consistent results on relative vasodilation were obtained for different vessels of the same volunteer (Fig. 4e) and across different 
volunteers (Fig. 4f), which indicates the general applicability of MB-L1 for assessing microvascular changes.

\section{Discussion}

Numerical simulations as well as phantom and in vivo experiments demonstrated the enhanced performance of the suggested MB-OAM framework with respect to standard OAM. This is in agreement with what has been shown in several OA tomographic configurations [38, 49-51]. However, OAM aims at a significantly higher resolution than OA tomography combined with large FOVs. The implementation of volumetric model-based iterative inversion methods is thereby significantly challenged by the fact that the full model matrix, corresponding to the linear operator mapping the initial pressure distribution (OA image) into measured pressure signals, becomes too large to be stored in GPU or even CPU memory. By exploiting the translational and axial symmetries of the model for the spherically focused transducer within the scanning plane and its limited lateral sensitivity, the model matrix was effectively "compressed" into a sub-matrix corresponding to a single scanning position and the voxel grids sufficiently close to it. The size of this sub-matrix is defined by the lateral sensitivity, the desired imaging depth, and the number of time instances, i.e., it is invariant with respect to an arbitrarily-large reconstruction grid. Therefore, the suggested model-based reconstruction framework is expected to be generally applicable in any OAM system. The advantages of model-based inversion stem from the fact that it accurately accounts for the broadband nature of OA signals. This is fundamentally different to other approaches considering an approximation of the transducer sensitivity field, which generally changes for different $O A$ sources due to the frequency dependence of the diffraction-limited acoustic focusing. In the current implementation, the model matrix was estimated theoretically by integrating the acoustic pressure distribution at the surface of the transducer, which represents a valid approximation for the curved thin PVDF film employed. However, the theoretical computation of the model matrix e.g. for transducers based on acoustic lenses or air-coupled transducers is more complex as it involves modeling acoustic interfaces $[52,53]$. The suggested reconstruction methodology can alternatively be used by experimentally estimating the model-matrix for a transducer position e.g. by raster-scanning a small particle or a light beam in the volume of interest [54]. Other parameters can also be considered in the model, such as the light fluence distribution or acoustic attenuation effects, which may lead to further improvements in quantification and spatial resolution $[55,56]$.

Acoustic diffraction has been considered as a limit for the achievable resolution in OA, similar to light diffraction in optical microscopy [57]. Super-resolution methods have massively impacted life sciences by smartly overcoming the optical diffraction limit [58]. The development of similar approaches enabling breaking through the acoustic diffraction barrier at depths not reachable with optical microscopy is also expected to highly impact the biomedical imaging field and boost the applicability of OA as a research and clinical tool. Well-established optical super-resolution methods can be taken as a reference for this purpose, although fundamental differences exist in physical principles and instrumentation. Recently, localization OA tomography (LOT) exploited the basic principle of photoactivated localization microscopy (PALM) to image beyond the resolution limit imposed by acoustic diffraction [9, 59-62]. The superresolution method suggested herein (MB-L1) has some analogies with stimulated emission depletion (STED) microscopy as it is based on a raster-scanning protocol where a non-linear response is induced. The non-linearity is however associated with the reconstruction procedure, hence MB-L1 also has 
similarities with super-resolution methods based on multiple observations with sub-pixel displacements and/or sparse image representations $[63,64]$. L1 has been shown to reduce the width of reconstructed structures in OA tomography $[65,66]$. Herein, we have further demonstrated that the suggested OA forward model is sufficiently accurate to better resolve closely separated sources - branching vascular structures - in vivo, which effectively demonstrates its performance as a super-resolution biomedical imaging tool. MB-L1 was further shown to provide accurate dimensional readings that can be exploited for the quantification of vasodilation or other microvascular alterations associated to human diseases.

In conclusion, we expect that the suggested MB-OAM framework significantly enhances the performance of this modality and enable the visualization of previously unresolvable structures beyond the acoustic diffraction barrier. The forward model accurately accounts for the transducer spatial response and the broadband nature of the induced pressure waves, which is essential for accurate reconstruction of $O A$ images. The fact that this could be achieved with low memory requirements and in a relatively short time anticipate its general applicability as a biomedical research tool and can further boost the translation of OAM as a label-free angiographic imaging method in the clinical setting.

\section{Methods}

\section{Forward modeling}

Excitation of OA signals in OAM is achieved with nanosecond laser pulses fulfilling thermal and stress confinement conditions. In an acoustically-uniform and non-attenuating medium, the time-resolved pressure signal $p(\boldsymbol{x}, t)$ at a point $\boldsymbol{x}$ is given as a function of absorbed optical energy density distribution $H\left(\boldsymbol{x}^{\prime}\right)$ as [67]

$$
p(\boldsymbol{x}, t)=\frac{\Gamma}{4 \pi c} \frac{\partial}{\partial t} \int_{S r(t)} \frac{H\left(\boldsymbol{x}^{\prime}\right)}{\left|\boldsymbol{x}-\boldsymbol{x}^{\prime}\right|} d S^{\prime}(t)
$$

where $\Gamma$ is the Grueneisen parameter, $c$ is the speed of sound, $\boldsymbol{x}^{r}$ is a point where light is absorbed, and $S^{\prime}(t)$ is a spherical surface defined as $\left|\boldsymbol{x}-\boldsymbol{x}^{\prime}\right|=c t$. The forward model in Eq. 1 was discretized with a two-step procedure - the time derivative was discretized using the finite difference method, and the surface integral was discretized using trilinear interpolation of neighboring voxel values [33]. Assuming a constant $\Gamma$ and $c$, the discrete forward model is then expressed (in arbitrary units) as the linear system of equations

$$
\boldsymbol{p}=\boldsymbol{A H},
$$

where $\boldsymbol{p}$ is a vector of pressure signals at all scanning positions, $\boldsymbol{A}$ is the model matrix and $\boldsymbol{H}$ is a vector of voxel values representing the absorbed energy density in the region of interest (ROI). The model matrix $\boldsymbol{A}$ describes the OA response to unit absorption at the voxel grid defined to cover the absorption distribution domain, and can be used to model the signals of a point detector with infinite bandwidth. More specifically, each column vector in $\boldsymbol{A}$ corresponds to one pair of voxel and scanning point. The OA signal collected by a finite-size transducer can be approximated by integrating pressure waves over the transducer surface [68]. This approximation represents the spatial impulse response (SIR) of the transducer, which can be modeled by dividing the transducer surface into sub-elements with position $\boldsymbol{x}_{\boldsymbol{j}}$ 
and area $\Delta \boldsymbol{x}_{j}$. The vector $s$ of signals collected by the transducer at all scanning positions is then expressed as

$$
\boldsymbol{s}=\sum_{j=1}^{M} \boldsymbol{p}_{x_{j}} \Delta x_{j}
$$

where $M$ is the number of sub-elements covering the transducer surface (Fig. 1c). Combining Eq. 2 and 3 , the discrete forward model becomes

$$
\boldsymbol{s}=\sum_{j=1}^{M} \boldsymbol{A}_{j} \Delta x_{j} \boldsymbol{H}=\boldsymbol{A}_{s} \boldsymbol{H} .
$$

Thereby, the model matrix $\boldsymbol{A}_{s}$ accounting for the SIR of the transducer is estimated as a weighted sum of $M$ model matrices corresponding to $M$ sub-elements.

Efficient reconstruction based on scanning symmetries

Model-based (MB) inversion involves minimizing an energy functional

$$
\boldsymbol{H}=\operatorname{argmin}_{H}\left\|\boldsymbol{s}_{m}-\boldsymbol{A}_{s} \boldsymbol{H}\right\|_{2}^{2}+\lambda R(\boldsymbol{H}),
$$

where $\boldsymbol{s}_{m}$ is the vector of measured signals, $R(\boldsymbol{H})$ is regularization term and $\lambda$ is the regularization parameter controlling the trade-off between regularization and data fidelity terms. The importance of $R(\boldsymbol{H})$ is two-fold - it makes the optimization procedure more robust to noise, and it incorporates apriori knowledge of the image $\boldsymbol{H}$. Herein, we considered the standard Tikhonov regularization based on the $L 2$ norm, where $R(\boldsymbol{H})$ is given by

$$
R(H)=\|H\|_{2}^{2},
$$

We refer to $M B$ inversion with Tikhonov regularization as the MB-L2 method. We also considered L1 regularization based on the $L 1$ norm, where $R(\boldsymbol{H})$ is given by

$$
R(\boldsymbol{H})=\|\boldsymbol{H}\|_{1} \text {. }
$$

We refer to $M B$ inversion with $L 1$ regularization as the MB-L1 method. The solution of Eq. 5 was computed iteratively with the LSQR algorithm [69] for MB-L2 and with the FISTA algorithm [70] for MBL1.

In both LSQR and FISTA algorithms, the most computationally demanding operations are the matrixvector multiplications $\boldsymbol{A}_{s} \boldsymbol{u}$ and $\boldsymbol{A}_{s}^{T} \boldsymbol{v}$, where $\boldsymbol{u}$ and $\boldsymbol{v}$ are updated in each iteration. The computational burden originates from the large-scale nature of OAM. This leads to a huge model matrix covering each pair of voxel and scanning position in the entire ROI, even if it is stored in sparse format [41]. However, since the spherically focused transducer barely senses voxels sufficiently distant in the lateral direction, the corresponding model matrix values were approximated as 0 , i.e., the model matrix only covers a $1 \mathrm{~mm}$ lateral extent (Fig. 1d), independently of an arbitrarily large ROI. The depth extent of model matrix scales with the collected dataset. Also, given that the transducer sensitivity field is translational- 
symmetric, the model vector is equivalent if the relative position between voxel and scanning point is maintained. Therefore, it was sufficient to generate the model matrix for a single scanning position, as it includes all unique lateral distances between pairs of voxel and scanning point. The "compressed" model matrix can then be pre-calculated in a reasonable time and stored in a memory-efficient way. To perform the matrix-vector multiplications, translational symmetries were considered to find the correspondences between voxels and model vectors (Fig. 1b). Specifically, we maintained two coordinate systems - an absolute coordinate system to index over the voxel grid, and a relative coordinate system, with the origin at each scanning position, to retrieve the corresponding model vector based on the lateral distance between a given voxel and the origin. The matrix-vector multiplications were parallelized and executed on GPU, which greatly improved computational speed.

\section{Numerical simulations}

The performance of the suggested MB-OAM methods, namely MB-L2 and MB-L1, was first tested on numerical simulations. In the first simulation, the absorption distribution within a $(2 \times 2 \times 3) \mathrm{mm}^{3} \mathrm{FOV}$ was modeled as four truncated paraboloids with diameters $60 \mu \mathrm{m}, 100 \mu \mathrm{m}, 150 \mu \mathrm{m}$, and $200 \mu \mathrm{m}$ (Fig. 1e), and a $25 \mu \mathrm{m}$ step size was used. The paraboloids were positioned $0.5 \mathrm{~mm}$ away from the center of the FOV in the lateral ( $x$ and $y$ ) directions, and equally separated within a $\pm 1 \mathrm{~mm}$ range from the acoustic focus in axial $(z)$ direction. The transducer surface was divided into 1200 sub-elements and the raw $O A$ signals at the center of each sub-element were calculated analytically [68]. The temporal sampling frequency was set to $250 \mathrm{MHz}$ for all simulations, consistent with the experimental setting. The $\mathrm{OA}$ signals were normalized to the maximum absolute value and $2 \%$ white Gaussian noise (standard deviation of 0.02) was added. MB-L2 and the standard SAFT [10] were used for image formation in this first simulation.

The second simulation was performed by considering two truncated paraboloids with $20 \mu \mathrm{m}$ diameter at the axial location of the acoustic focus for a FOV of $(200 \times 200 \times 150) \mu m^{3}$ and $5 \mu \mathrm{m}$ step size. The paraboloids were first separated by $60 \mu \mathrm{m}$ in the $\mathrm{x}$ direction to ensure they can be resolved by all three methods in comparison - SAFT, MB-L2, and MB-L1 (Fig. 2a, left). Subsequently, they were separated by $40 \mu \mathrm{m}$ in the $\mathrm{x}$ direction, which was empirically demonstrated to be beyond the acoustic diffraction limit for the filters employed (Fig. 2a, right). Specifically, the raw signals and the model matrix were bandpass filtered between $0.5 \mathrm{MHz}$ and $80 \mathrm{MHz}$, yielding a diffraction-limited resolution of $61 \mu \mathrm{m}$ [43]. The $\mathrm{OA}$ signals were normalized by the maximum absolute value and no noise was added.

A third simulation was performed to validate the capability of MB-L1 to accurately quantify micro-vessel dimensions. To this end, $\mathrm{OA}$ signals corresponding to truncated paraboloid absorbers with diameters of $20,30,45,60,80$, and $100 \mu \mathrm{m}$ were simulated, and independently inverted with both MB-L2 and MB-L1. The simulated absorbers were positioned at the depth of the acoustic focus and at the center of a FOV covering a $(250 \times 250 \times 250) \mu m^{3}$ region with $5 \mu \mathrm{m}$ step size. The OA signals were normalized to the maximum absolute value and $2 \%$ white Gaussian noise was added.

OAM set-up 
A recently proposed burst-mode OAM system was used [11] (Suppl. Fig. 1). The system employs a custom-made, $9 \mu \mathrm{m}$ foil PVDF spherically focused transducer ( $7 \mathrm{~mm}$ focal distance and $0.43 \mathrm{NA}$ ). Three laser sources at $532 \mathrm{~nm}, 578 \mathrm{~nm}$, and $1064 \mathrm{~nm}$ were combined and coupled into a multimode fiber, which was guided through a central hole $(0.9 \mathrm{~mm}$ diameter) of the transducer, thus providing concentric illumination and acoustic detection. Two of the laser sources have fixed wavelengths at $532 \mathrm{~nm}$ and $1064 \mathrm{~nm}$ (Onda $532 \mathrm{~nm}$ and $1064 \mathrm{~nm}$, Bright Solutions, UK), and the third source is a tunable dye laser (Credo, Sirah Lasertechnik, Germany) with wavelength set to $578 \mathrm{~nm}$. The transducer was translated laterally in the x-y plane above the sample (Fig. 1a) to acquire a time-resolved OA signal at each scanning position. Specifically, a fast-moving scanning stage moves constantly forth and back between the boundaries of the previously defined scan window while monitoring its position. Following each predefined incremental stage movement, all three lasers were triggered in a cascade with a $6 \mu$ s delay in between. Motion artifacts were avoided by averting acceleration and deceleration at each scanning position. The system thus enables rapid acquisition of multi-wavelength volumetric datasets over large FOV. In all experiments, the temporal sampling frequency was set to 250 MSPS and no signal averaging was performed.

\section{Phantom experiments}

The suggested $M B$ inversion framework was tested on two phantom experiments. A first phantom was used to test if MB-L1 is able to break the acoustic diffraction limit on experimental data. This consisted of a single micro-sphere with $20 \mu \mathrm{m}$ diameter embedded in $1.3 \%(\mathrm{w} / \mathrm{v})$ agar. The phantom was placed with the micro-sphere at the axial location of the acoustic focus and a scan covering a ( $200 \times 200 \times 150)$ $\mu m^{3}$ FOV was performed with $5 \mu \mathrm{m}$ step size. The $532 \mathrm{~nm}$ laser source was used, with up to $2.5 \mathrm{kHz}$ pulse repetition rate (PRR) and $50 \mu \mathrm{J}$ per-pulse energy (PPE). The single micro-sphere image in the phantom was first individually formed. Subsequently, the signal volume was artificially shifted by $40 \mu \mathrm{m}$ in the $x$ direction and super-imposed with the original volume, which is equivalent to the signal volume of two micro-spheres separated by the same distance.

A second phantom consisting of four carbon fibers with $7 \mu \mathrm{m}$ diameter embedded in $1.3 \%(\mathrm{w} / \mathrm{v})$ agar was imaged. The purpose of this phantom experiment was to assess the overall performance of SAFT and the suggested $\mathrm{MB}$ reconstruction methods to reconstruct elongated structures. The scan covered a $(1 \times 2 \times 1) \mathrm{mm}^{3} \mathrm{FOV}$ with $10 \mu \mathrm{m}$ step size. The PRR was again limited to $2.5 \mathrm{kHz}$ and the PPE of the $532 \mathrm{~nm}$ laser was set to $50 \mu \mathrm{J}$.

In vivo mouse skin imaging

The in vivo imaging performance of the suggested $M B$ inversion framework was tested by imaging dorsal mouse skin. To this end, a CD-1 mouse ( 8 weeks old; Charles River Laboratories, Germany) was anesthetized with isoflurane, and placed in prone position on a heating pad. The back skin was shaved and cleaned before imaging. A scan covering a $10 \times 30 \mathrm{~mm}^{2}$ FOV with a $25 \mu \mathrm{m}$ step size was performed, giving a volumetric dataset at each wavelength $(532 \mathrm{~nm}, 578 \mathrm{~nm}$, and $1064 \mathrm{~nm})$. The PRR was limited to up to $2.5 \mathrm{kHz}$ and the PPEs for the three lasers were set to $25 \mu \mathrm{J}, 25 \mu \mathrm{J}$, and $100 \mu \mathrm{J}$, respectively. Before reconstruction, the raw signals were bandpass filtered between $1 \mathrm{MHz}$ and $120 \mathrm{MHz}$, and further 
median filtered with kernel size of $3 \times 3 \times 3$. The animal experiment was performed in accordance with the Swiss Federal Act on Animal Protection and were approved by the Canton Veterinary Office Zurich.

Image visualization and vessel quantification

Visualization of the dorsal skin vasculature was done using maximum intensity projections (MIPs) along all Cartesian dimensions. Volume rendering was performed in Paraview 5.8.0 [71]. The images of the mouse skin vasculature were shown with color-coded depth. This was done by multiplying a background map (reconstructed volume), a foreground map (3-channel color encoder), and a transparency value. Foreground colors were set to blue, green, and orange from deeper to shallower regions. The number of vessels was quantified with a previously reported automatic vessel analysis and segmentation (AVSA) algorithm [48]. In short, reconstructed images were binarized, skeletonized, and vessel center-lines fitted by a spline. Vessel edges were found by computing the image gradient along the normal direction of the central line. To quantify the number of vessels achieved in SAFT, MB-L2 and MB-L1, two signal volumes acquired at $532 \mathrm{~nm}$ and $578 \mathrm{~nm}$ from the multi-wavelength in vivo dataset were used. Each of the two volumes were further split into half along the y direction, which increases the sample size and gives a total of four individual datasets. Reconstructed vessels in an upper-right sub-region were individually visualized based on branch point, central line and edge (Fig. 3e). The number of vessels in images reconstructed with SAFT, MB-L2 and MB-L1 was plotted, and the mean value was noted for all three methods (Fig. 3f).

\section{Optical angiographic imaging}

The in vivo performance of the suggested MB-OAM image formation framework was validated against wide-field optical imaging. To this end, the dorsal skin of an athymic nude-Fox1nu mouse (20 week-old; Envigo RMS R.V., Netherlands) was sequentially imaged with different modalities. The mouse was anesthetized with isoflurane ( $3 \% \mathrm{v} / \mathrm{v}$ for induction, $1.5 \% \mathrm{v} / \mathrm{v}$ for maintenance) in a mixture of air and oxygen with flow rates $0.8 \mathrm{~L} / \mathrm{min}$ and $0.2 \mathrm{~L} / \mathrm{min}$, respectively. The mouse was placed in prone position on a heating pad maintaining body temperature during the experiment. First, a USB microscope (Dinolite digital microscope, Taiwan) was used to take a bright field image of the mouse. Subsequently, epifluorescence images were taken in the first and second near infrared windows (NIR-I and NIR-II). NIR-I imaging was performed after tail-vein injection of $100 \mu \mathrm{L}$ indocyanine green (ICG, Sigma-Aldrich Chemie $\mathrm{GmbH}$, Switzerland) at a concentration of $1 \mathrm{mg} / \mathrm{ml}$. The fluorescence image was recorded with an EMCCD camera (iXon Life, Andor, UK) under $700 \mathrm{~nm}$ excitation. NIR-II imaging was performed following tail-vein injection of $50 \mu \mathrm{L}$ NIR-II quantum dots emitting at $1600 \mathrm{~nm}$ (NBDY-0018, Nirmidas Biotech, USA) at a concentration of $5 \mathrm{mg} / \mathrm{ml}$. Time-lapse fluorescence images were recorded with a commercial SWIR camera (WiDy SenS 640V-ST, NiT, France) under $855 \mathrm{~nm}$ excitation. An OAM image was also acquired with $532 \mathrm{~nm}$ excitation wavelength and $25 \mu \mathrm{m}$ step size, covering a FOV of $10 \times 30 \mathrm{~mm}^{2}$. A big vertical vessel in the middle of the mouse back was selected as an anatomical landmark for comparison of the different modalities. All animal experiments were performed in accordance with the Swiss Federal Act on Animal Protection and were approved by the Canton Veterinary Office Zurich. 
Human nailfold imaging

Nailfold imaging in three healthy volunteers was performed to demonstrate the general applicability of the suggested MB-OAM methodology in humans. All volunteers were informed and gave written consent to participate in the experiments. Dilation and constriction of the microvasculature in this region was achieved with a thermal stimulation protocol consisting of two main steps [72]. First, the hand of the volunteer was immersed in cold water $\left(\sim 15^{\circ} \mathrm{C}\right)$ for 5 minutes. Three consecutive scans of the index finger of this hand, each taking approximately 1.5 minutes, were performed immediately after immersion. Subsequently, the same hand was immersed in warm water $\left(\sim 40^{\circ} \mathrm{C}\right)$ for 5 minutes and the scans were repeated immediately after. All scans were performed at $532 \mathrm{~nm}$ and $10 \mu \mathrm{m}$ step size, covering a $4 \times 3 \mathrm{~mm}^{2} \mathrm{FOV}$. The PPE and the PRR of the laser were set to $25 \mu \mathrm{J}$ and $2 \mathrm{kHz}$, respectively.

Images of the entire FOV were first reconstructed with MB-L2. To this end, OA signals were divided into different frequency bands [73]. Specifically, band-pass filters with cut-off frequencies $10-20 \mathrm{MHz}, 20-$ $30 \mathrm{MHz}, \quad 30-40 \mathrm{MHz}$ and $40-60 \mathrm{MHz}$ were applied. OA signals in each frequency band were independently used for reconstruction considering a regularization parameter of 1000 and 5 iterations. A weighted sum (weighting factors of $0.45,0.4,0.1$, and 0.05 from low to high frequency bands) of the reconstructed images was then calculated. Images of a smaller FOV $\left(1.6 \times 0.8 \mathrm{~mm}^{2}\right)$ at the junction of nail and skin were formed with MB-L1. To this end, OA signals were band-pass filtered with cut-off frequencies 20-25 MHz. Profiles corresponding to different microvessels were extracted from the 3D images to assess the dilation from cold to warm stimulus. The width of the corresponding vessels was estimated via Gaussian fitting of the profiles. Differences were assessed for different vessels of the same volunteer and across volunteers. The MB-L2 and MB-L1 images were visualized as MIPs along the $z$ (depth) dimension, while the depth information was further color-coded from orange to cyan, corresponding to shallow and deep regions.

\section{Author contributions}

XLDB and DR conceived the concept and devised the study. WL, UH, AO, YG, and XLDB developed the reconstruction framework and implemented the reconstruction code. UH developed the imaging system. UH and WL performed all experiments. WL performed the reconstructions and data analysis. QZ and ZC performed the optical imaging experiments. JR implemented depth-encoding visualization and vessel quantification code. DR and XLDB supervised the work. All authors contributed to writing and revising the manuscript.

\section{Acknowledgments}

This work was supported by the Helmut Horten Stiftung (Project Deep Skin, X.L.D.B.) and by the European Research Council under grant agreement ERC-2015-CoG-682379 (D.R.).

\section{Data and code availability}


Data generated during this study and code used to process images are available from the corresponding author upon reasonable request.

\section{Ethics declarations}

The authors declare no conflicts of interests.

a

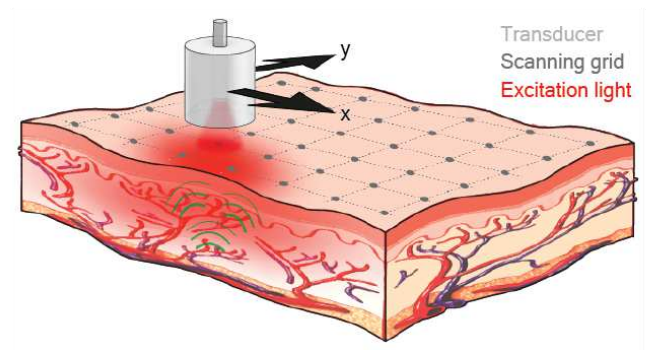

b

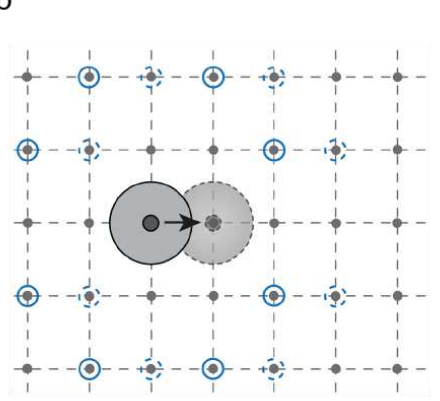

d

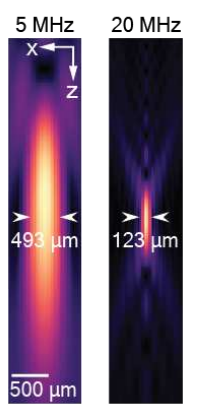

C

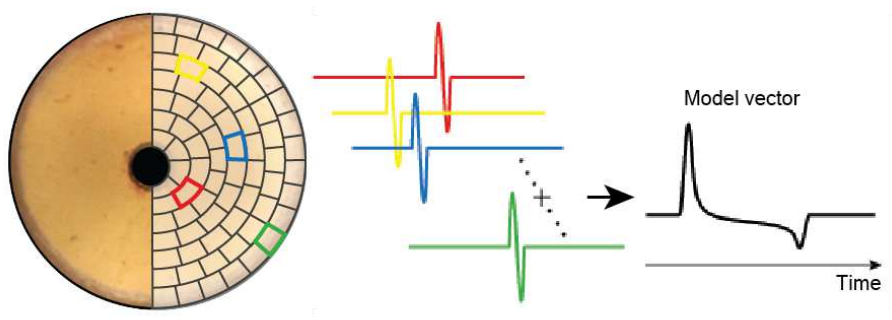

e

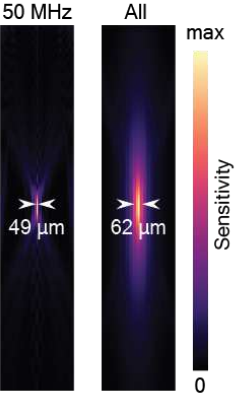

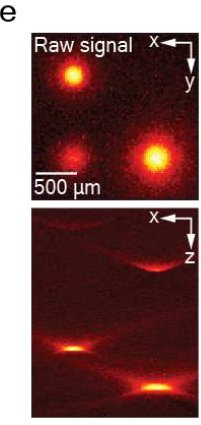

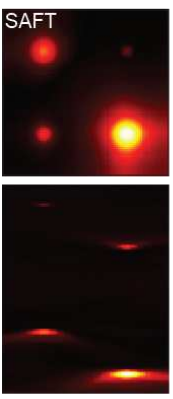

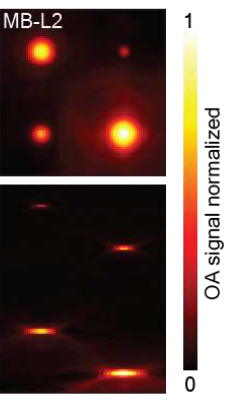

Fig. 1 Time-domain model for OAM. (a) Lay-out of the raster-scan scanning protocol. (b) Schematic representation of the scanning symmetries. The OA signals generated at all voxels labeled with blue circles for the indicated transducer position are equivalent. Signals are preserved if the transducer and voxels are shifted by the same distance (dashed lines). (c) Actual photograph of the transducer active area (left) along with a representation of the OA model based on discretizing transducer surface into sub-elements (right). The OA signals corresponding to a given voxel and all surface elements are superimposed to estimate the collected time-resolved signal for that voxel. (d) Cross-sectional view of the amplitude spectral density of the modelled time-resolved signals for three different frequencies along with amplitude of these signals in the time domain. FWHM are indicated. (e) OAM images reconstructed by superimposing the acquired signals (left), with the SAFT method (middle) and with the MB-L2 method (right). 
a

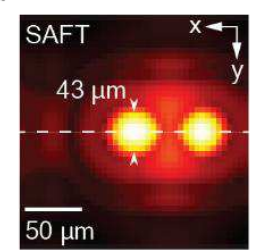

MB-L2

$41 \mu \mathrm{m}$

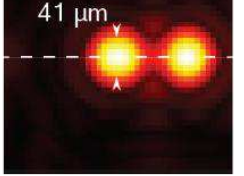

MB-L1

$13 \mu \mathrm{m}_{\mathrm{v}}$

$-13 \mu m_{r}$

$x^{x}$

b

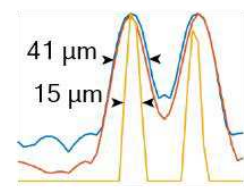

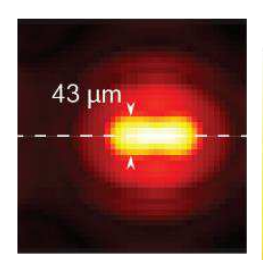
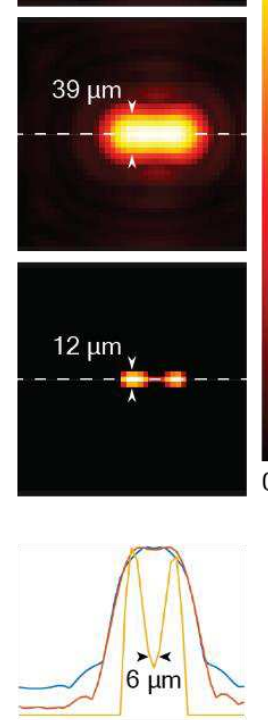

C
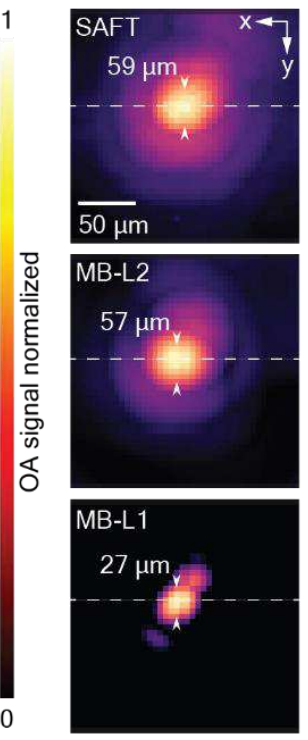

d

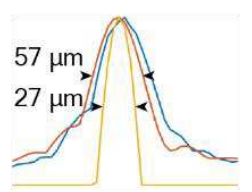

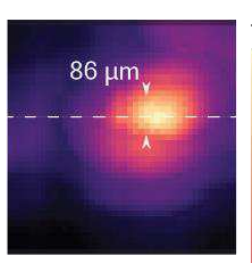
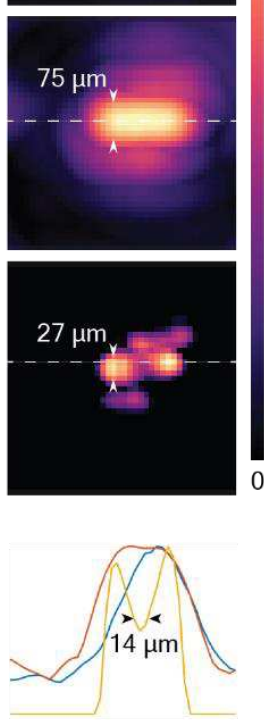

e
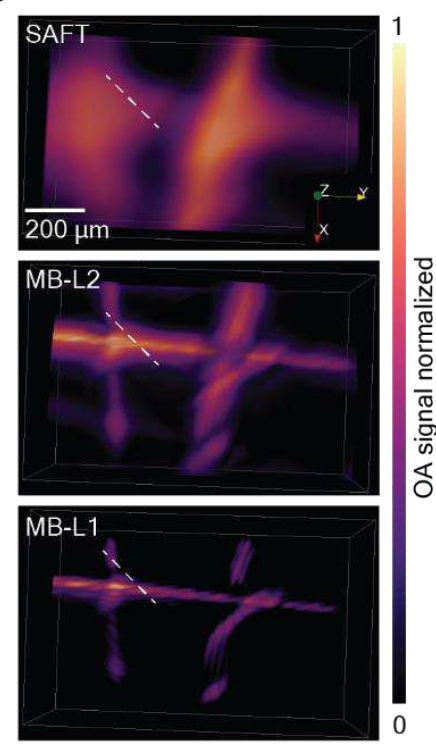

$\mathrm{f}$

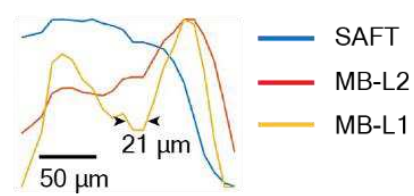

Fig. 2 Super-resolution imaging beyond the acoustic diffraction limit. (a) MIPs of the OAM images reconstructed with the SAFT (top), MB-L2 (middle) and MB-L1 (bottom) methods from simulated data corresponding to two $20 \mu \mathrm{m}$ absorbers separated by $60 \mu \mathrm{m}$ (left) and $40 \mu \mathrm{m}$ (right). FWHM are indicated. (b) Cross-sectional profiles for the dashed lines indicated in (a). FWHM are indicated in the left panel, and separation for points $>20 \%$ of the minimum is indicated in the right panel. (c) MIPs of the OAM images reconstructed with the SAFT (top), MB-L2 (middle) and MB-L1 (bottom) methods from experimental data corresponding to a $20 \mu \mathrm{m}$ sphere. Reconstructions from the original collected timeresolved signals and from the superposition of signals shifted by $40 \mu \mathrm{m}$ are shown (left and right respectively). FWHM are indicated. (d) Cross-sectional profiles for the dashed lines indicated in (c). FWHM are indicated in the left panel and separation for points $>20 \%$ of the minimum is indicated in the right panel. (e) MIPs of the OAM images reconstructed with the SAFT (top), MB-L2 (middle) and MB-L1 (bottom) methods from experimental data corresponding to $7 \mu \mathrm{m}$ carbon fibers. (f) Cross-sectional profiles for the dashed lines indicated in (e). Separation for points $>20 \%$ of the minimum is indicated. 
a
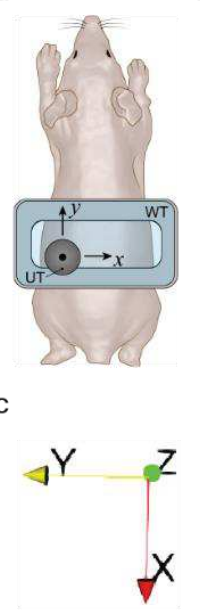

- Branch poin

- Central line

--- Edge

$\stackrel{-\cdots}{-=} \cdot$ Vessel

b
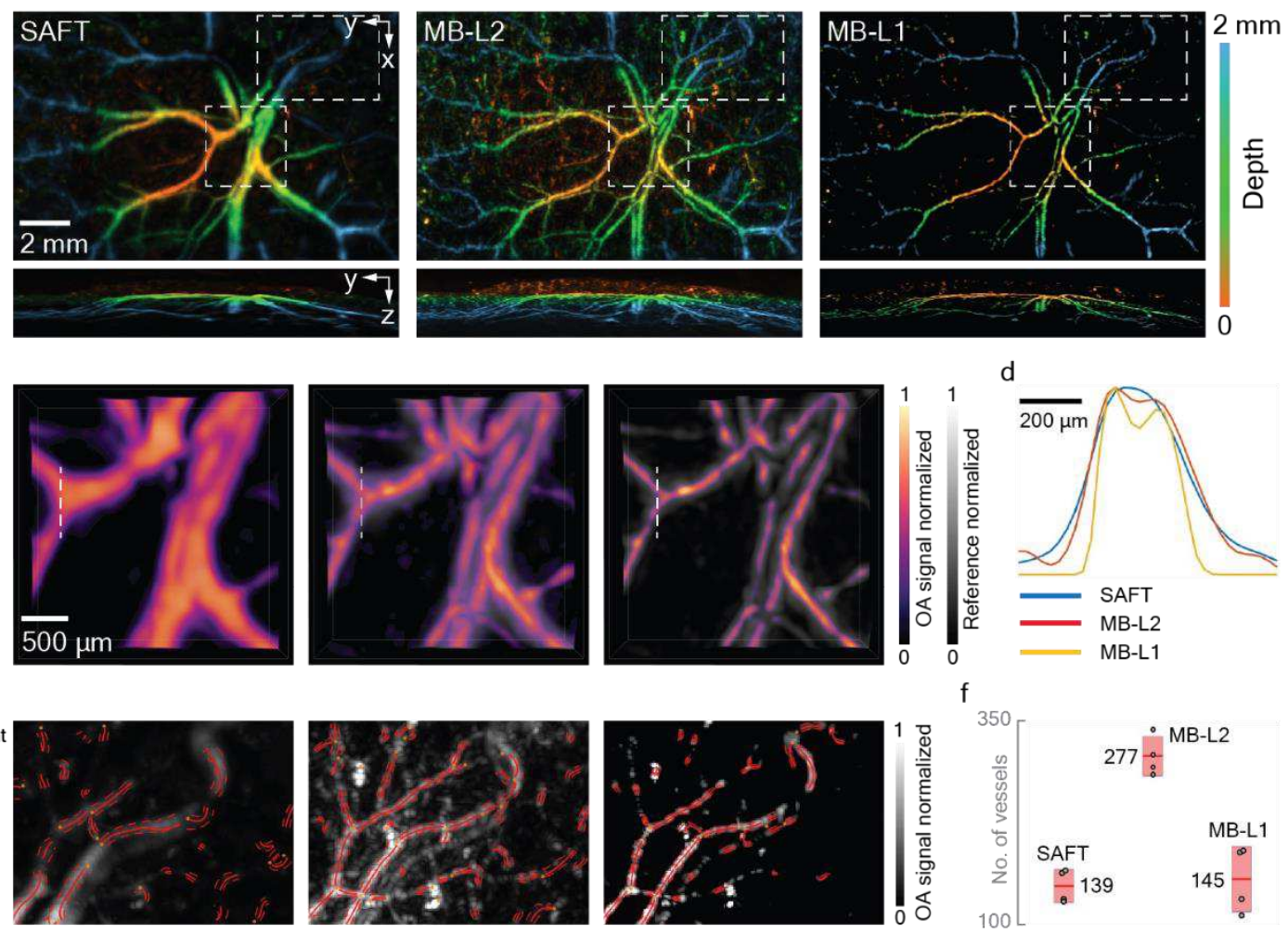

Fig. 3 Enhanced angiographic imaging capabilities. (a) Lay-out of the in vivo imaging scheme indicating the scanned region. (b) Depth color-coded MIPs of the OAM images reconstructed with the SAFT (left), MB-L2 (middle) and MB-L1 (right) methods. (c) Three-dimensional views of the central region indicated in (b). The reference images are the SAFT image in the middle panel and the MB-L2 image in the right panel. (d). Cross-sectional profiles indicated in (c). (e) Reconstructed vessels in the binarized image corresponding to the indicated region in the upper right corner in (b). (f) Number of detected vessels for four different in vivo datasets reconstructed with SAFT, MB-L2 and MB-L1. The average number of vessels are indicated for each reconstruction method. 
a

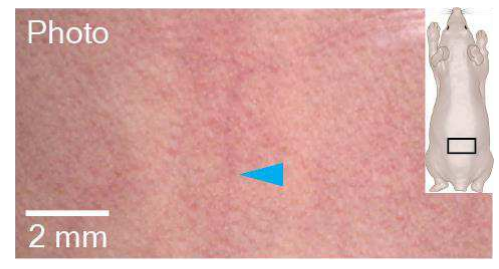

NIR-I

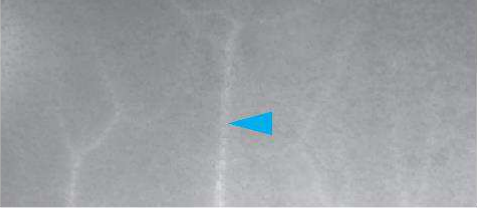

NIR-II
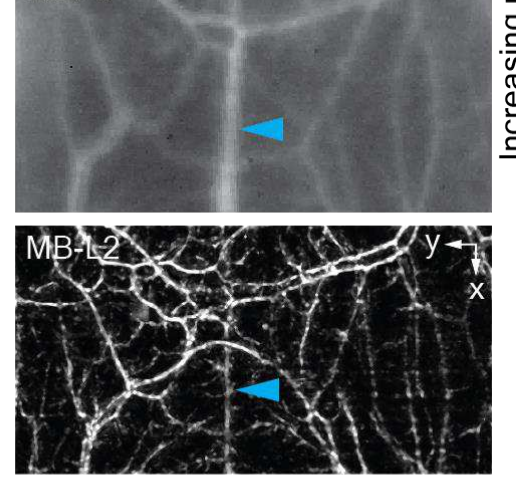

OA/FL signal (a.u.) b
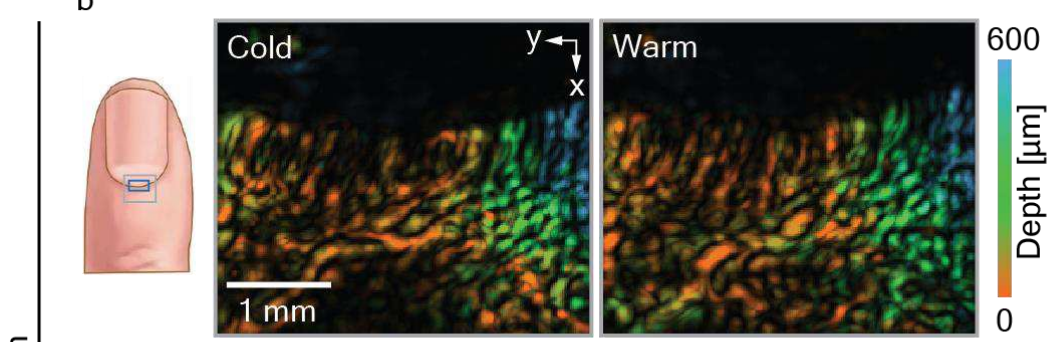

C

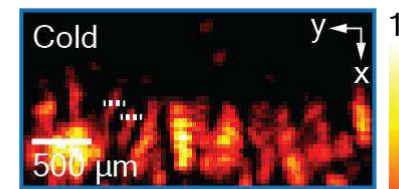

\section{Warm}
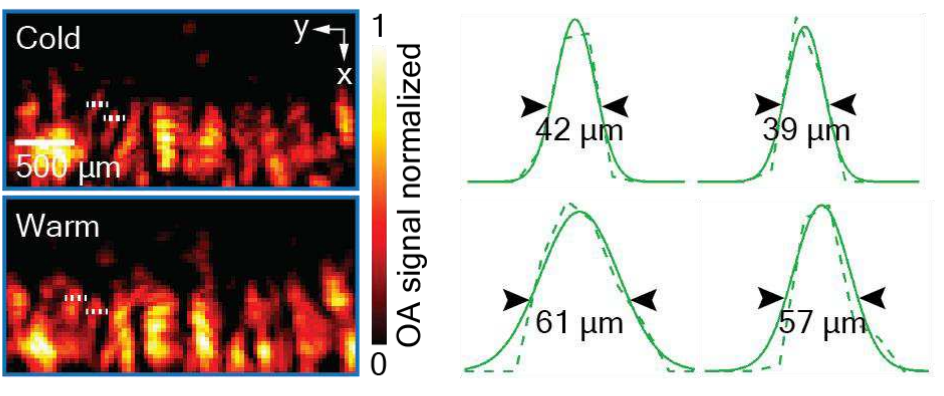

e

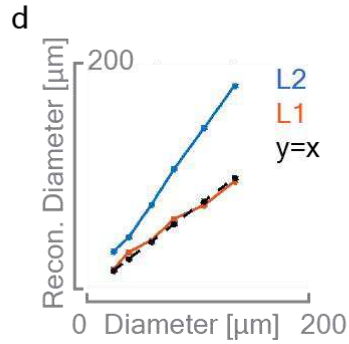

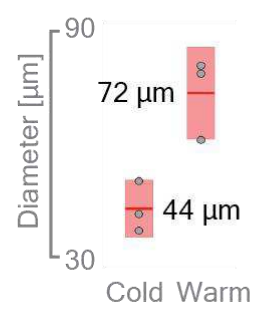

f

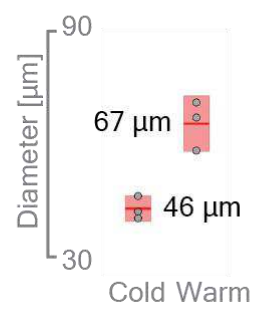

Fig. 4 Validation and quantification of microvascular changes. (a) Comparison of OAM with established angiographic imaging approaches. From top to bottom - bright field optical imaging, NIR-I fluorescence image following injection of ICG, NIR-II fluorescence image following injection of QDs, OAM image reconstructed with MB-L2. The imaged region in the mouse is indicated. (b) OAM images (reconstructed with MB-L2) of the cuticle vasculature of a healthy volunteer following immersion in cold and warm water. The imaged region in the finger is indicated. (c) OAM images (reconstructed with MB-L1) of the nailfold arterioles and venules. The imaged region in the finger is indicated. The indicated profiles and fitted Gaussian curves are also shown. (d) Reconstructed diameter of a simulated sphere as a function of the actual diameter for MB-L1 and MB-L2. The line corresponding to ideal reconstruction $(y=x)$ is indicated. (e) Measured diameters (FWHMs in the MB-L1 images) of 3 microvessels in a volunteer following immersion in cold and warm water. (f) Averaged measured diameters (FWHMs in the MB-L1 images) in $n=3$ healthy volunteers following immersion in cold and warm water. 


\section{References}

1. Wang, L.V. and J. Yao, A practical guide to photoacoustic tomography in the life sciences. Nature methods, 2016. 13(8): p. 627.

2. Deán-Ben, X., et al., Advanced optoacoustic methods for multiscale imaging of in vivo dynamics. Chemical Society Reviews, 2017. 46(8): p. 2158-2198.

3. Taruttis, A. and V. Ntziachristos, Advances in real-time multispectral optoacoustic imaging and its applications. Nature photonics, 2015. 9(4): p. 219-227.

4. Li, L., et al., Single-impulse panoramic photoacoustic computed tomography of small-animal whole-body dynamics at high spatiotemporal resolution. Nature biomedical engineering, 2017. 1(5): p. 1-11.

5. Deán-Ben, X.L., et al., Spiral volumetric optoacoustic tomography visualizes multi-scale dynamics in mice. Light: Science \& Applications, 2017. 6(4): p. e16247-e16247.

6. Deán-Ben, X.L., H. López-Schier, and D. Razansky, Optoacoustic micro-tomography at 100 volumes per second. Scientific reports, 2017. 7(1): p. 1-8.

7. Zhang, H.F., et al., Functional photoacoustic microscopy for high-resolution and noninvasive in vivo imaging. Nature biotechnology, 2006. 24(7): p. 848-851.

8. Jathoul, A.P., et al., Deep in vivo photoacoustic imaging of mammalian tissues using a tyrosinase-based genetic reporter. Nature Photonics, 2015. 9(4): p. 239-246.

9. Kim, J., et al., Super-resolution localization photoacoustic microscopy using intrinsic red blood cells as contrast absorbers. Light: Science \& Applications, 2019. 8(1): p. 1-11.

10. Park, S., et al., Acoustic resolution photoacoustic microscopy. Biomedical Engineering Letters, 2014. 4(3): p. 213-222.

11. Hofmann, U.A., et al., Rapid functional optoacoustic micro-angiography in a burst mode. Optics Letters, 2020. 45(9): p. 2522-2525.

12. Omar, M., et al., Pushing the optical imaging limits of cancer with multi-frequency-band rasterscan optoacoustic mesoscopy (RSOM). Neoplasia, 2015. 17(2): p. 208-214.

13. Aguirre, J., et al., Precision assessment of label-free psoriasis biomarkers with ultra-broadband optoacoustic mesoscopy. Nature Biomedical Engineering, 2017. 1(5): p. 1-8.

14. Haedicke, K., et al., High-resolution optoacoustic imaging of tissue responses to vasculartargeted therapies. Nature Biomedical Engineering, 2020. 4(3): p. 286-297.

15. Jeon, M., J. Kim, and C. Kim, Multiplane spectroscopic whole-body photoacoustic imaging of small animals in vivo. Medical \& biological engineering \& computing, 2016. 54(2-3): p. 283-294.

16. Li, X., et al., Optoacoustic mesoscopy analysis and quantitative estimation of specific imaging metrics in Fitzpatrick skin phototypes II to V. Journal of biophotonics, 2019. 12(9): p. e201800442.

17. Estrada, H., et al., Real-time optoacoustic brain microscopy with hybrid optical and acoustic resolution. Laser Physics Letters, 2014. 11(4): p. 045601.

18. Rebling, J., et al., Dual-wavelength hybrid optoacoustic-ultrasound biomicroscopy for functional imaging of large-scale cerebral vascular networks. Journal of biophotonics, 2018. 11(9): p. e201800057.

19. Liu, W., et al., Quad-mode functional and molecular photoacoustic microscopy. Scientific reports, 2018. 8(1): p. 1-10.

20. Li, M.-L., et al., Improved in vivo photoacoustic microscopy based on a virtual-detector concept. Optics letters, 2006. 31(4): p. 474-476.

21. Stepinski, T. and F. Lingvall. Synthetic aperture focusing techniques for ultrasonic imaging of solid objects. in 8th European Conference on Synthetic Aperture Radar. 2010. VDE. 
22. Turner, J., et al., Universal weighted synthetic aperture focusing technique (W-SAFT) for scanning optoacoustic microscopy. Optica, 2017. 4(7): p. 770-778.

23. Spadin, F., et al., Quantitative comparison of frequency-domain and delay-and-sum optoacoustic image reconstruction including the effect of coherence factor weighting. Photoacoustics, 2020. 17: p. 100149.

24. Park, J., et al., Delay-multiply-and-sum-based synthetic aperture focusing in photoacoustic microscopy. Journal of biomedical optics, 2016. 21(3): p. 036010.

25. Mozaffarzadeh, M., et al., Enhanced contrast acoustic-resolution photoacoustic microscopy using double-stage delay-multiply-and-sum beamformer for vasculature imaging. Journal of biophotonics, 2019. 12(11): p. e201900133.

26. $\mathrm{Xu}, \mathrm{M}$. and L.V. Wang, Universal back-projection algorithm for photoacoustic computed tomography. Physical Review E, 2005. 71(1): p. 016706.

27. Treeby, B.E. and B.T. Cox, $k$-Wave: MATLAB toolbox for the simulation and reconstruction of photoacoustic wave fields. Journal of biomedical optics, 2010. 15(2): p. 021314.

28. Jin, H., et al., Fast and high-resolution three-dimensional hybrid-domain photoacoustic imaging incorporating analytical-focused transducer beam amplitude. IEEE transactions on medical imaging, 2019. 38(12): p. 2926-2936.

29. Aguirre, J., et al., A low memory cost model based reconstruction algorithm exploiting translational symmetry for photoacoustic microscopy. Biomedical optics express, 2013. 4(12): p. 2813-2827.

30. Yao, L., L. Xi, and H. Jiang, Photoacoustic computed microscopy. Scientific reports, 2014. 4: p. 4960.

31. Deán-Ben, X.L., et al. Three-dimensional modeling of the transducer shape in acoustic resolution optoacoustic microscopy. in Photons Plus Ultrasound: Imaging and Sensing 2014. 2014. International Society for Optics and Photonics.

32. Mitsuhashi, K., K. Wang, and M.A. Anastasio, Investigation of the far-field approximation for modeling a transducer's spatial impulse response in photoacoustic computed tomography. Photoacoustics, 2014. 2(1): p. 21-32.

33. Ding, L., X.L. Deán-Ben, and D. Razansky, Efficient 3-D model-based reconstruction scheme for arbitrary optoacoustic acquisition geometries. IEEE Transactions on Medical Imaging, 2017. 36(9): p. 1858-1867.

34. Drozdov, G., A. Levi, and A. Rosenthal, The Impulse Response of Negatively Focused Spherical Ultrasound Detectors and Its Effect on Tomographic Optoacoustic Reconstruction. IEEE transactions on medical imaging, 2019. 38(10): p. 2326-2337.

35. Arridge, S., et al., Accelerated high-resolution photoacoustic tomography via compressed sensing. Physics in Medicine \& Biology, 2016. 61(24): p. 8908.

36. Frikel, J. and M. Haltmeier, Efficient regularization with wavelet sparsity constraints in photoacoustic tomography. Inverse Problems, 2018. 34(2): p. 024006.

37. Ding, L., et al., Constrained inversion and spectral unmixing in multispectral optoacoustic tomography. IEEE transactions on medical imaging, 2017. 36(8): p. 1676-1685.

38. Hauptmann, A., et al., Model-based learning for accelerated, limited-view 3-d photoacoustic tomography. IEEE transactions on medical imaging, 2018. 37(6): p. 1382-1393.

39. Matthews, T.P., et al., Parameterized joint reconstruction of the initial pressure and sound speed distributions for photoacoustic computed tomography. SIAM journal on imaging sciences, 2018. 11(2): p. 1560-1588.

40. Özbek, A., X.L. Deán-Ben, and D. Razansky, Optoacoustic imaging at kilohertz volumetric frame rates. Optica, 2018. 5(7): p. 857-863. 
41. Ding, L., D. Razansky, and X.L. Deán-Ben, Model-based reconstruction of large three-dimensional optoacoustic datasets. IEEE Transactions on Medical Imaging, 2020.

42. Shan, T., et al., GPU-based acceleration and mesh optimization of finite-element-method-based quantitative photoacoustic tomography: a step towards clinical applications. Applied optics, 2017. 56(15): p. 4426-4432.

43. Yao, J. and L.V. Wang, Photoacoustic microscopy. Laser \& photonics reviews, 2013. 7(5): p. 758778.

44. Rosenthal, A., V. Ntziachristos, and D. Razansky, Acoustic inversion in optoacoustic tomography: A review. Current Medical Imaging, 2013. 9(4): p. 318-336.

45. Hansen, P.C., Discrete inverse problems: insight and algorithms. 2010: SIAM.

46. Davoudi, N., X.L. Deán-Ben, and D. Razansky, Deep learning optoacoustic tomography with sparse data. Nature Machine Intelligence, 2019. 1(10): p. 453-460.

47. Arridge, S.R., et al., On the adjoint operator in photoacoustic tomography. Inverse Problems, 2016. 32(11): p. 115012.

48. Estrada, H., et al., Intravital optoacoustic and ultrasound bio-microscopy reveal radiationinhibited skull angiogenesis. Bone, 2020. 133: p. 115251.

49. Lutzweiler, C., X.L. Deán-Ben, and D. Razansky, Expediting model-based optoacoustic reconstructions with tomographic symmetries. Medical physics, 2014. 41(1): p. 013302.

50. Lou, Y., et al., Analysis of the use of unmatched backward operators in iterative image reconstruction with application to three-dimensional optoacoustic tomography. IEEE Transactions on Computational Imaging, 2019. 5(3): p. 437-449.

51. Han, Y., et al., Three-dimensional optoacoustic reconstruction using fast sparse representation. Optics letters, 2017. 42(5): p. 979-982.

52. Deán-Ben, X.L., et al., Non-contact optoacoustic imaging with focused air-coupled transducers. Applied Physics Letters, 2015. 107(5): p. 051105.

53. Ma, H., et al., Noncontact photoacoustic angiography with an air-coupled ultrasonic transducer for evaluation of burn injury. Applied Physics Letters, 2019. 114(13): p. 133701.

54. Seeger, M., et al., Pushing the boundaries of optoacoustic microscopy by total impulse response characterization. Nature Communications, 2020. 11(1): p. 1-13.

55. Bu, S., et al., Model-based reconstruction integrated with fluence compensation for photoacoustic tomography. IEEE transactions on biomedical engineering, 2012. 59(5): p. 13541363.

56. Brochu, F.M., et al., Towards quantitative evaluation of tissue absorption coefficients using light fluence correction in optoacoustic tomography. IEEE transactions on medical imaging, 2016. 36(1): p. 322-331.

57. Abbe, E., Beiträge zur Theorie des Mikroskops und der mikroskopischen Wahrnehmung. Archiv für mikroskopische Anatomie, 1873. 9(1): p. 413-468.

58. Sigal, Y.M., R. Zhou, and X. Zhuang, Visualizing and discovering cellular structures with superresolution microscopy. Science, 2018. 361(6405): p. 880-887.

59. Vilov, S., B. Arnal, and E. Bossy, Overcoming the acoustic diffraction limit in photoacoustic imaging by the localization of flowing absorbers. Optics letters, 2017. 42(21): p. 4379-4382.

60. Dean-Ben, X.L. and D. Razansky, Localization optoacoustic tomography. Light: Science \& Applications, 2018. 7(4): p. 18004-18004.

61. Zhang, P., et al., In vivo superresolution photoacoustic computed tomography by localization of single dyed droplets. Light: Science \& Applications, 2019. 8(1): p. 1-9.

62. Deán-Ben, X.L., et al., Noninvasive three-dimensional optoacoustic localization microangiography of deep tissues. arXiv preprint arXiv:2007.00372, 2020. 
63. Li, X., et al., A multi-frame image super-resolution method. Signal Processing, 2010. 90(2): $\mathrm{p}$. 405-414.

64. Yang, J., et al., Image super-resolution via sparse representation. IEEE transactions on image processing, 2010. 19(11): p. 2861-2873.

65. Egolf, D.M., R.K. Chee, and R.J. Zemp, Sparsity-based reconstruction for super-resolved limitedview photoacoustic computed tomography deep in a scattering medium. Optics letters, 2018. 43(10): p. 2221-2224.

66. Deán-Ben, X.L., et al., Acoustic scattering mediated single detector optoacoustic tomography. Physical review letters, 2019. 123(17): p. 174301.

67. Cox, B.T., et al., k-space propagation models for acoustically heterogeneous media: Application to biomedical photoacoustics. The Journal of the Acoustical Society of America, 2007. 121(6): $p$. 3453-3464.

68. Queirós, D., et al., Modeling the shape of cylindrically focused transducers in three-dimensional optoacoustic tomography. Journal of biomedical optics, 2013. 18(7): p. 076014.

69. Paige, C.C. and M.A. Saunders, LSQR: An algorithm for sparse linear equations and sparse least squares. ACM Transactions on Mathematical Software (TOMS), 1982. 8(1): p. 43-71.

70. Beck, A. and M. Teboulle, A fast iterative shrinkage-thresholding algorithm for linear inverse problems. SIAM journal on imaging sciences, 2009. 2(1): p. 183-202.

71. Ayachit, U., The paraview guide: a parallel visualization application. 2015: Kitware, Inc.

72. Plumb, A.A., et al., Rapid volumetric photoacoustic tomographic imaging with a Fabry-Perot ultrasound sensor depicts peripheral arteries and microvascular vasomotor responses to thermal stimuli. European radiology, 2018. 28(3): p. 1037-1045.

73. Aguirre, J., et al., Assessing nailfold microvascular structure with ultra-wideband raster-scan optoacoustic mesoscopy. Photoacoustics, 2018. 10: p. 31-37. 


\section{Supplementary Files}

This is a list of supplementary files associated with this preprint. Click to download.

- Li20210AMSupplementaryFigures.docx 